\title{
BMJ Open Role of the total progressive motile sperm count (TPMSC) in different infertility factors in IUI: a retrospective cohort study
}

\author{
Haiyan Lin (1) , ${ }^{1}$ Yu Li, ${ }^{1}$ Songbang Ou, ${ }^{1}$ Xuedan Jiao, ${ }^{1}$ Wenjun Wang, ${ }^{1}$ \\ Peter Humaidan, ${ }^{2}$ Qingxue Zhang ${ }^{1}$
}

To cite: Lin H, Li Y, Ou S, et al. Role of the total progressive motile sperm count (TPMSC) in different infertility factors in IUI: a retrospective cohort study. BMJ Open 2021;11:e040563. doi:10.1136/ bmjopen-2020-040563

- Prepublication history and additional material is published online only. To view please visit the journal online (http://dx.doi. org/10.1136/bmjopen-2020040563).

Received 27 May 2020 Revised 26 November 2020 Accepted 02 December 2020

Check for updates

(c) Author(s) (or their employer(s)) 2021. Re-use permitted under CC BY-NC. No commercial re-use. See rights and permissions. Published by BMJ.

${ }^{1}$ Reproductive center, Department of Obestetrics and Gynecology, Sun YatSen University, Sun Yat-Sen Memorial Hospital, Guangzhou, China

${ }^{2}$ The Fertility Clinic, Skive Regional Hospital, Skive, Faculty of Health, Aarhus University, Denmark

Correspondence to

Qingxue Zhang;

Zhqingx@mail.sysu.edu.cn

\section{ABSTRACT}

Objective The objective of this retrospective cohort study was to explore the optimal range of the total progressive motile sperm count (TPMSC) for live birth in couples with varying infertility diagnosis undergoing intrauterine insemination (IUI) in a university-affiliated teaching hospital.

Methods A total of 2647 couples and $5171 \mathrm{IUI}$ cycles were included between January 2015 and December 2018. Of those, 1542 cycles were performed due to unexplained infertility, 1228 cycles due to anovulation, 1120 cycles due to mild male factor infertility and 122 cycles due to mild endometriosis. The primary outcome measure was live birth rate (LBR). The secondary outcome measure was clinical pregnancy rate (CPR).

Results The CPR and LBR were highest in patients with a diagnosis of anovulation compared with the other three groups of patients. The CPR and LBR in patients with unexplained, mild male factor and mild endometriosis were comparable. For the patients with mild male factor infertility, the CPR with prewash TPMSC of $>75.0 \mathrm{M}$ and postwash TPMSC of $65.10 \mathrm{M}$ was above $10 \%$, statistically significantly higher than other quartiles of TPMSC $(p<0.05)$. The LBR with postwash TPMSC of $>65.10 \mathrm{M}$ was statistically significantly higher than other groups $(p<0.05)$. However, in patients with unexplained infertility, the CPR and LBR were not statistically different in quartiles of TPMSC, being less than $10 \%$. Overall, there was only one clinical pregnancy and no live birth in patients $>40$ years of age.

Conclusions In conclusion, the infertility diagnosis plays a significant role for the patient undergoing IUI. Thus, the anovulatory patients benefitted most from IUI, irrespective of TPMSC. For patients with unexplained infertility, TPMSC does not affect the success rate of IUI. Overall,female patients more than 40 years old should not be referred to IUI.

\section{INTRODUCTION}

Intrauterine insemination (IUI) is mainly indicated for mild male factor infertility, unexplained infertility, mild endometriosis as well as oligo-ovulation/anovulation. Despite its widespread use in daily clinical practice, the relatively low clinical pregnancy rate
Strengths and limitations of this study

- This study has a large sample size to explore the intrauterine insemination (IUI) success rate in different infertility factors.

- It is found that the anovulatory patients benefitted most from IUI, irrespective of the total progressive motile sperm count (TPMSC) of $\geq 10 \mathrm{M} / \mathrm{mL}$. For the unexplained infertile patients, TPMSC has little relationship with IUI success rate.

- This study is a retrospective analysis from a single centre, and other factors related with IUI success were not considered.

(CPR) after IUI is still a matter of debate. The large population-based data from the European Society of Human Reproduction and Embryology showed a delivery rate per IUI cycle of $8.3 \%,{ }^{1}$ and the latest national report on ART(Assisted Reproductive Technology) status in mainland China showed a delivery rate of $10.5 \%(6.7 \%-14.3 \%)$ per IUI cycle. ${ }^{2}$ From the latest observational retrospective study, IUI is more cost- effective in delivering one live birth than IVF(In Vitro Fertilization), and is associated with lower risk of maternal and neonatal complications. ${ }^{3}$ As this is a huge data analysis, does IUI perform the same in patients with different infertility factors? The question to be answered is which group of patients will benefit the most from IUI treatment?

Male factor infertility is currently increasing and the clinical diagnosis is only based on semen parameters. ${ }^{4}$ A recent publication based on a longitudinal cohort study concluded that the prewash total motile sperm count (TMSC) had a better correlation with the natural conception ongoing pregnancy rate than the WHO 2010 classification. ${ }^{5}$ Thus far, only a few studies evaluated the predictive value of the $\mathrm{WHO}$ criteria for semen 
in infertile couples. ${ }^{5-12}$ Moreover, insemination motile sperm count of more than one million is considered as an important predictive parameter in IUI. ${ }^{79}$ Regardless of this, there is still insufficient evidence to conclude that IUI is effective for mild male factor infertility. ${ }^{13}$

In another line, unexplained infertility is an unpredictable state without any definitive and demonstrable causes of infertility. A common diagnosis of unexplained infertility includes normal standard investigations for tubal patency, ovulation assessment and semen analysis; and unexplained infertility accounts for up to $30 \%$ of infertility. ${ }^{14}$ The first-line treatment for the unexplained infertile patient is still debated, ${ }^{14-16}$ and in 2013, the National Institute for Health and Clinical Excellence guideline recommended IVF for unexplained infertility couples who did not conceive spontaneously after 2 years of regular unprotected sexual intercourse. ${ }^{17}$ This recommendation could be considered quite aggressive for some patients and physicians, resulting in the fact that the guideline is poorly adhered to worldwide. ${ }^{17-19} \mathrm{~A}$ few studies reported that the duration of infertility, secondary infertility and sperm motility were associated with IUI success rate ${ }^{7-9}$ 20-26; however, in patients with unexplained infertility, as the cost-efficiency between IUI and IVF has ever been proved ${ }^{27}$ high-quality studies exploring factors that determine IUI success in unexplained infertility are needed.

In the present analysis, we explored the optimal total progressive motile sperm count (TPMSC) for live birth rate (LBR) beyond $10 \%$ in patients with mild male infertility, unexplained infertility, oligo-ovulation/anovulation, and mild endometriosis.

\section{MATERIALS AND METHODS \\ Study population}

This study is a retrospective cohort study using data from our local hospital database. All infertile couples undergoing IUI at the Reproductive Centre, Sun Yat-sen Memorial Hospital of Sun Yat-sen University from January 2015 to December 2018 were included. Cycles without complete information or cancelled IUI cycles were excluded. Infertile couples were considered suitable for IUI, based on a semen analysis and patency examination of the fallopian tubes. Semen analysis parameters included in the analysis were obtained on the day of insemination. Couple characteristics including age, body mass index (BMI), duration of infertility, female baseline follicle-stimulating hormone $(\mathrm{FSH})$, prewash and postwash semen concentrations, and motile rate were collected. Couples with unexplained infertility (ovulatory, normal semen analysis and with evidence of bilateral patent tubes), anovulation, mild male factor (semen analyses with one only of the semen parameters below the normal range refers to $\mathrm{TSC} \geq 15 \mathrm{M} / \mathrm{mL}$, and $\mathrm{PR} \geq 32 \%$, normal morphological forms $>4 \%)$ and mild endometriosis-related infertility (diagnosed with mild endometriosis by laparoscopy or sole small endometriosis cyst $<4 \mathrm{~cm}$ ) were analysed separately. The primary outcome was LBR.

\section{Patient and public involvement}

No patient was involved in this retrospective analysis of data.

\section{Semen preparation}

After 3-7 days of abstinence, the semen was collected about 2 hours before insemination. Semen was examined according to the WHO guidelines (the fifth edition) ${ }^{28}$ and prepared using the density-gradient centrifugation method. The details are as follows: (1) use two liquids, SpermGrade and SpermRinse, to prepare a gradient solution containing 90\% SpermGrade and $45 \%$ SpermGrade in advance; (2) the gradient solution is equilibrated to room temperature; (3) using a sterile Pasteur pipette, add $1.0-2.0 \mathrm{~mL}$ of $90 \%$ gradient liquid to the sterile, sharp-bottomed centrifuge tube as the "lower layer liquid' according to the volume of the semen, and gently add an equal volume of $45 \%$ gradient liquid above the liquid surface as the 'upper layer liquid'; (4) suction the completely liquefied semen with a sterile Pasteur pipette and slowly add it to a $15 \mathrm{~mL}$ conical centrifuge tube with gradient centrifugal fluid, and centrifuge at $500 \mathrm{~g}$ for $15 \mathrm{~min}$; (5) discard the supernatant, add the precipitate to $2 \mathrm{~mL}$ G-IVF ${ }^{\mathrm{TM}}$ PLUS fertilization fluid(Vitrolife,Sweden), and centrifuge at $200 \mathrm{~g}$ for 4-10 min; and (6) aspirate the supernatant and leave $0.5 \mathrm{~mL}$ of sperm suspension for IUI use. The insemination was carried out by physicians, only.

The prewash TPMSC was calculated by multiplying the total volume and sperm concentration by the progressive motility percentage. The postwash TPMSC was determined by multiplying $0.5 \mathrm{~mL}$ for insemination and sperm concentration by the progressive motility percentage. Morphology was not used for calculations and analysis.

\section{IUI treatment}

Ovarian stimulation protocol

If the patient had an irregular or anovulatory menstrual cycle, the ovarian stimulation cycle was preferred. Ovarian stimulation protocols included clomiphene citrate (CC; CodalSynto, Cyprus), CC in combination with gonadotropins, letrozole (Hengrui Medicine, China), letrozole in combination with gonadotropins, or gonadotropins only. Treatment with CC, letrozole or gonadotropins alone started on the fifth day of the cycle for a total of 5 days. Treatment with gonadotropins, in combination with CG or letrozole, started after completion of the oral agents and continued until the desired follicular response. A urine LH (Luteinizing Hormone) test was performed daily by the patient once the dominant follicle reached $14 \mathrm{~mm}$ in diameter. Insemination was performed either on the urine LH test-positive day or on the day after trigger with HCG 5000-10 000 U (uHCG(urinary Human Chorionic Gonadotropin), Livzon, China, or rHCG(recombinant Human Chorionic Gonadotropin), 
Merck,USA) in patients with urine LH-negative testing, once the dominant follicle reached a size of $18 \mathrm{~mm}$.

The goal for all patients with IUI was one to three dominant follicles (diameter $\geq 14 \mathrm{~mm}$ ). Most cycles had one to two dominant follicles; very few cases had three dominant follicles. If the number of dominant follicles was more than three, the cycles were cancelled or converted to IVF.

\section{Natural cycle}

If the patient had a regular menstrual cycle and underwent her first cycle, natural cycle IUI was preferred. Ultrasound monitoring started on the 10-12th day, combined with urine LH testing once the dominant follicle reached $14 \mathrm{~mm}$ in diameter. When the diameter of the dominant follicle reached $18 \mathrm{~mm}$, IUI was performed on the urine LH test-positive day or the day after trigger with Human Chorionic Gonadotropin 5000-10 $000 \mathrm{U}$ in patients with urine LH-negative testing.

\section{Insemination}

A soft catheter was used for insemination and the patient had bed rest for $30 \mathrm{~min}$ after the IUI procedure. About 2 days later, ovulation was confirmed by transvaginal ultrasound, followed by oral dydrogesterone supplementation (Duphaston, Abbott Healthcare, USA) $10 \mathrm{mg}$ two times per day for 2 weeks until a urine pregnancy test was performed.

\section{Measurements}

Baseline characteristics such as age, duration of infertility, BMI, female baseline FSH and the semen parameters of prewash and postwash were analysed.

\section{Reproductive outcomes}

Clinical pregnancy was defined by transvaginal ultrasonographic visualisation of one or more gestational sacs or definitive clinical signs of pregnancy. Clinical pregnancy also included ectopic pregnancy. Live birth was defined as a viable delivery beyond 28 weeks' gestation.

\section{Statistical analysis}

All data were tested by one sample Kolmogorov-Smirnov test to confirm normality. Categorical variables were assessed by $\chi^{2}$ test or Fisher's exact test as appropriate. Non-normality distribution data was analysed by a nonparametric test (Mann-Whitney U test and Kruskal-Wallis test) such as age and TPMSC. Binary logistic regression was performed to gain associated factors with live birth. Receiver operating characteristic (ROC) curves were calculated to determine the predictive value between prewash/postwash TPMSC and the clinical pregnancy and live birth. A p value of $<0.05$ was considered statistically significant. IBM SPSS Statistics V.22 was used to analyse data.

\section{RESULTS}

A total of 5361 IUI cycles, all homologous inseminations, were included in the analysis. Of those, a total of 133 cycles were excluded, including 69 cycles converted to IVF due to multifollicular development (more than three dominant follicles), 5 couples (cycles) who refused to undergo insemination due to poor postwash TPMSC and 51 cycles in which the man could not provide an ejaculate on the IUI day; moreover, eight cycles were cancelled due to other factors such as fever. Finally, 57 cycles were excluded due to incomplete data. Finally, a total cohort of 5171 IUI cycles performed in 2647 couples was included for analysis. According to the infertility diagnosis, there were 1542 cycles (29.8\%) of unexplained infertility, 1228 cycles $(23.7 \%)$ of anovulation and 122 cycles $(2.4 \%)$ of mild endometriosis and 1120 cycles $(21.7 \%)$ of mild male factor. To obtain as 'clean' as possible a sample size, 1159 cycles $(22.4 \%)$ with other factors such as multiple factors, mild pelvic (mild adhesions during laparoscopy) and tubal factors were not included in this study.

\section{Baseline characteristics}

The baseline characteristics of recruited couples are shown in table 1 . The median age in couples with unexplained infertility or mild male factor infertility was statistically significantly higher than that of anovulatory couples (female age: 31 vs $29, \mathrm{p} \leq 0.001$; male age: 33 vs $32, \mathrm{p} \leq 0.001)$. Moreover, the female BMI was significantly higher in patients with anovulation factor infertility.

\section{Prewash and postwash TPMSC}

The prewash and postwash TPMSCs were statistically lower in patients with mild male factor than patients with unexplained infertility ( $36 \mathrm{M}$ vs $60 \mathrm{M}, 34.76 \mathrm{M}$ vs $54 \mathrm{M}$; $\mathrm{p} \leq 0.001)$. The CPR and LBR were highest in patients with anovulation compared with the other three groups. In contrast, the CPR and LBR in patients with unexplained, mild male factor and mild endometriosis were comparable (see table 1 ). Overall, the live birth rate per cycle was $8.5 \%(342 / 4003)$. For women $>40$ years of age, there was only one pregnancy in 78 cycles (CPR 1.3\%) and no live birth.

\section{Reproductive outcomes}

To explore the possible role of prewash and postwash TPMSC in patients with different infertility diagnosis, the quartiles of TPMSC and, accordingly, CPR and LBR are shown in figure 1. It appears that the CPR and LBR in unexplained infertility are not statistically correlated with the prewash and postwash TPMSC. Importantly, the CPR and LBR in patients with unexplained infertility are not higher than $10 \%$. Patients with anovulation factor benefitted most from IUI, the CPR and LBR being higher than $10 \%$, and not associated with prewash and postwash TPMSCs.

For mild male factor infertility, the CPRs with prewash TPMSC $>75 \mathrm{M}$ and postwash TPMSC $>65.10 \mathrm{M}$ were above $10 \%$, statistically significantly higher than other quartiles of TPMSC $(p<0.05)$. The LBR in postwash TPMSC $>65.10$ $\mathrm{M}$ was statistically significantly higher than other quartiles $(p<0.05)$. In patients with mild endometriosis, the 
Table 1 Characteristics, CPR and LBR in IUI

\begin{tabular}{|c|c|c|c|c|c|}
\hline Items & Unexplained infertility & Anovulation factor & Mild endometriosis & Mild male factor & P value \\
\hline Cycles, n (\%) & $1542(29.8)$ & $1228(23.7)$ & $122(2.4)$ & $1120(21.7)$ & \\
\hline Female age (years) & $31(29-34)$ & $29(27-32)$ & $30(28-33)$ & $31(29-34)$ & $<0.001$ \\
\hline Duration of infertility (years) & $3(2-5)$ & $3(2-5)$ & $3(2-5)$ & $3(2-5)$ & $<0.001$ \\
\hline Female baseline FSH (U/L) & 7.34 (6.26-8.67) & $6.8(5.78-8.04)$ & 7.08 (5.94-8.91) & $7.36(6.22-8.71)$ & $<0.001$ \\
\hline Prewash TPMSC (M) & $60(30-108)$ & $54.2(25.7-97.3)$ & $54(30-90)$ & $36(15-75)$ & $<0.001$ \\
\hline Postwash TPMSC (M) & $54(27-90)$ & $49.8(26.1-84.7)$ & $54(27-81)$ & $34.76(11.25-65.10)$ & $<0.001$ \\
\hline CPR, \% (n) & $8.0(124)$ & $18.0(221)$ & $7.4(9)$ & $8.4(94)$ & $<0.001$ \\
\hline LBR, \% (n) & $5.4(83 / 1538)$ & $14.5(177 / 1224)$ & $6.6(8 / 122)$ & $6.6(74 / 1119)$ & $<0.001$ \\
\hline
\end{tabular}

Kruskal-Wallis test were used for non-normality distribution data, results expressed as median (quartile range).

$\mathrm{BMI}$, body mass index; CPR, clinical pregnancy rate; FSH, follicle-stimulating hormone; IUI, intrauterine insemination; LBR, live birth rate; TPMSC, total progressive motile sperm count.

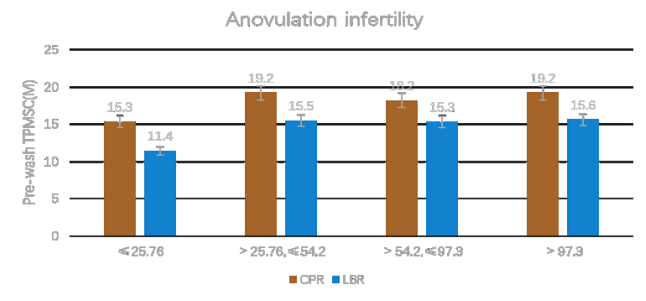

Mild endometriosis
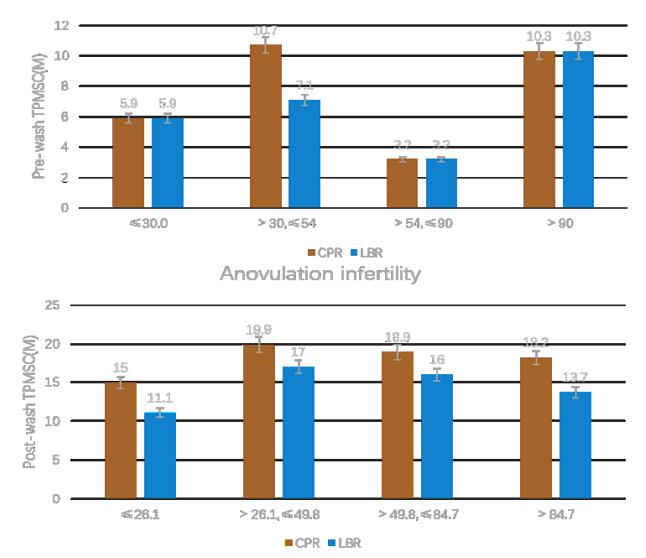

Mild endometriosis

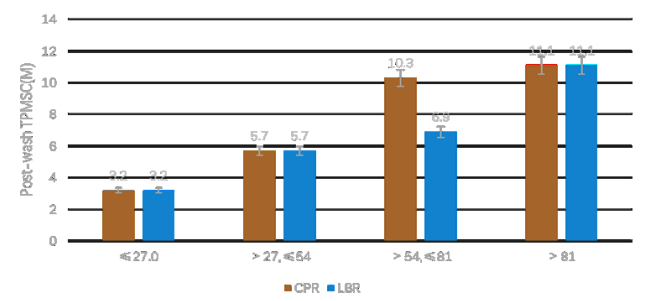

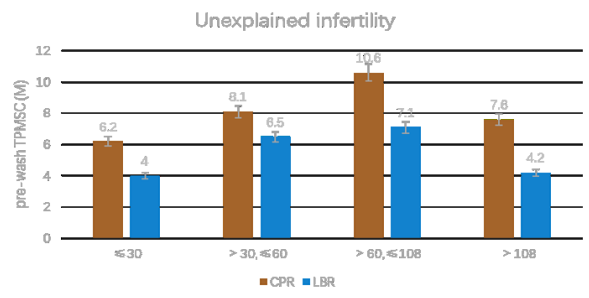

Mild male infertility

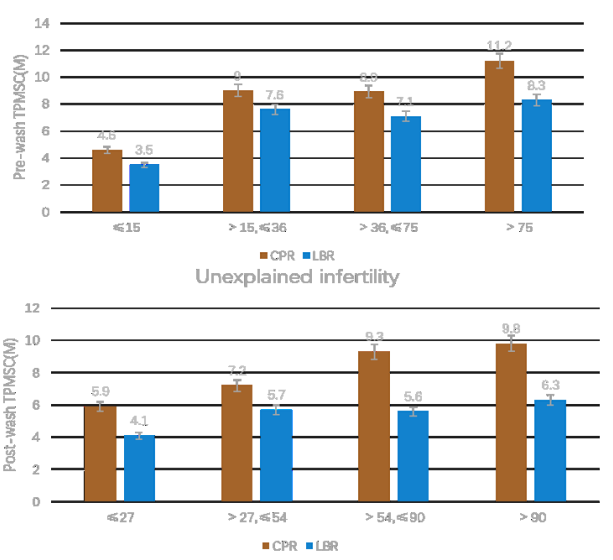

Mild male infertility

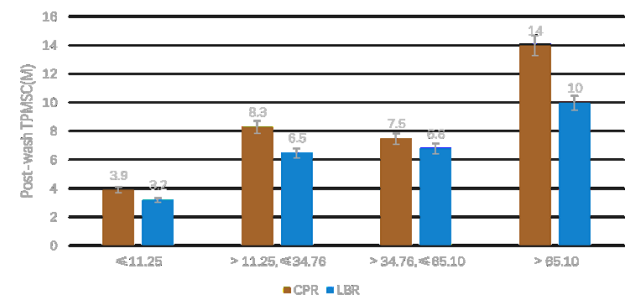

Figure 1 CPR and LBR in quartiles of prewash and postwash TPMSC according to groups with different infertility factors. (AD) CPRs and LBRs in different quartile groups of prewash TPMSC. (E-H) showed the CPR and LBR in different quartiles groups of postwash TPMSC. The blue bars represent the CPR. The orange bar represents LBR. CPR, clinical pregnancy rate; LBR, live birth rate; TPMSC, total progressive motile sperm count. 
Table 2 Comparison of characteristics between live birth and non-live birth cycles in mild male infertility couples

\begin{tabular}{|c|c|c|c|}
\hline & Live birth cycles & $\begin{array}{l}\text { Non-live birth } \\
\text { cycles }\end{array}$ & $\begin{array}{l}P \\
\text { value }\end{array}$ \\
\hline Cycles, n (\%) & 74 & 1045 & \\
\hline Female age (years) & $30.39 \pm 3.29$ & $31.47 \pm 4.02$ & 0.025 \\
\hline Male age (years) & $32.62 \pm 3.78$ & $33.71 \pm 4.70$ & 0.02 \\
\hline $\begin{array}{l}\text { Duration of } \\
\text { infertility (years) }\end{array}$ & $3.40 \pm 2.10$ & $3.58 \pm 2.10$ & 0.469 \\
\hline $\begin{array}{l}\text { Female baseline } \\
\text { FSH (U/L) }\end{array}$ & $7.30 \pm 2.43$ & $7.66 \pm 2.22$ & 0.19 \\
\hline $\begin{array}{l}\text { Female BMI (kg/ } \\
\left.\mathrm{m}^{2}\right)\end{array}$ & $21.09 \pm 2.43$ & $20.65 \pm 2.57$ & 0.16 \\
\hline Male BMI $\left(\mathrm{kg} / \mathrm{m}^{2}\right)$ & $24.86 \pm 3.80$ & $25.50 \pm 5.12$ & 0.922 \\
\hline $\begin{array}{l}\text { Prewash TPMSC } \\
\text { (M) }\end{array}$ & 48 (20-90) & $36(15-75)$ & 0.063 \\
\hline $\begin{array}{l}\text { Postwash TPMSC } \\
\text { M) }\end{array}$ & $46.5(19.65-83.70)$ & $32.55(10.23-63.0)$ & 0.002 \\
\hline
\end{tabular}

BMI, body mass index; FSH, follicle-stimulating hormone; TPMSC, total progressive motile sperm count.

CPR and LBR with prewash TPMSC $>90 \mathrm{M}$ and postwash TPMSC $>54 \mathrm{M}$ were above $10 \%$-higher than other groups-but without being statistically significant.

The live birth cycles were compared with non-live birth cycles in mild male infertility couples. We found the female age, male age and postwash TPMSC were significantly different in live birth cycles (table 2). After adjusting for the age of the couple, binary multivariate logistic regression showed that postwash TPMSC was statistically associated with live birth in patients with mild male factor infertility, despite the OR $(1.010,95 \%$ CI 1.002 to $1.017 ; \mathrm{p}=0.012$ ) being very low (see table 3 ).

A ROC cure was performed in patients with mild male factor infertility to explore the predictive value of prewash and postwash TPMSCs in clinical pregnancy and live birth. It proves that postwash TPMSC was better than prewash TPMSC to predict clinical pregnancy and live birth according to the area under the curve (CP: 0.621, $95 \%$ CI 0.564 to 0.677 , vs $0.578,95 \%$ CI 0.520 to 0.635 ; LB: $0.605,95 \%$ CI 0.543 to 0.667 , vs $0.565,95 \%$ CI 0.502 to 0.628 ). Based on the Youden index, the cut-off of postwash TPMSC was $55.40 \mathrm{M}$ for clinical pregnancy and 15.37 $\mathrm{M}$ for live birth, and the predictive sensitivity was $50 \%$ and $86.5 \%, 71 \%$ and $31 \%$ in specificity (see figure 2 ).

\section{DISCUSSION}

In this large analysis, we found that for patients with mild male factor infertility, with prewash TPMSC $>75.0 \mathrm{M}$ and postwash TPMSC $>65.10 \mathrm{M}$, the CPR was above $10 \%$ and LBR reached the reported average level. In patients with unexplained infertility, the CPR and LBR were less than $10 \%$, and in the patients with anovulation infertility, the CPR and LBR were highest (beyond $10 \%$ ) in IUI cycles regardless of TPMSC. Overall, the LBR (8.5\%) was consistent with the previous reported level, and there was only one clinical pregnancy and no live births in women aged $>40$ years.

Table 3 Binary multivariate logistic regression of characteristics associated with live birth

\begin{tabular}{|c|c|c|c|c|c|c|}
\hline & \multirow{2}{*}{$\begin{array}{l}\text { Unexplained infertility } \\
\text { OR }(95 \% \mathrm{CI})\end{array}$} & \multirow[b]{2}{*}{$P$ value } & \multirow{2}{*}{$\begin{array}{l}\text { Anovulation factor } \\
\text { OR }(95 \% \mathrm{Cl})\end{array}$} & \multirow[b]{2}{*}{$P$ value } & \multirow{2}{*}{$\begin{array}{l}\text { Mild male factor infertility } \\
\text { OR }(95 \% \mathrm{Cl})\end{array}$} & \multirow[b]{2}{*}{ P value } \\
\hline & & & & & & \\
\hline Female age (years) & 0.973 (0.856 to 1.108$)$ & 0.68 & $0.944(0.865$ to 1.031$)$ & 0.2 & 0.907 (0.791 to 1.040$)$ & 0.16 \\
\hline$>30, \leq 37$ & $2.985(0.758$ to 11.755$)$ & 0.12 & 0.805 (0.166 to 3.913$)$ & 0.79 & 2.752 (0.319 to 23.784$)$ & 0.36 \\
\hline$>37$ & 1 & 0.24 & 1 & 0.62 & 1 & 0.29 \\
\hline Duration of infertility (years) & $1.002(0.797$ to 1.261$)$ & 0.98 & $1.045(0.862$ to 1.266$)$ & 0.66 & 0.977 (0.719 to 1.326$)$ & 0.88 \\
\hline$\leq 3$ & $1.363(0.347$ to 5.344$)$ & 0.66 & 2.578 (0.859 to 7.733$)$ & 0.09 & 1.098 (0.201 to 6.009$)$ & 0.91 \\
\hline$>3, \leq 5$ & $1.238(0.452$ to 3.386$)$ & 0.68 & $1.852(0.837$ to 4.098$)$ & 0.13 & 0.973 (0.299 to 3.162) & 0.96 \\
\hline$>5$ & 1 & 0.9 & 1 & 0.24 & 1 & 0.96 \\
\hline Female baseline FSH (U/L) & 1.021 (0.873 to 1.194$)$ & 0.8 & 0.942 (0.844 to 1.052$)$ & 0.29 & 0.878 (0.745 to 1.034$)$ & 0.12 \\
\hline Female BMI $\left(\mathrm{kg} / \mathrm{m}^{2}\right)$ & 1.099 (0.960 to 1.258$)$ & 0.17 & $1.014(0.922$ to 1.115$)$ & 0.77 & 1.154 (1.011 to 1.316$)$ & 0.03 \\
\hline Male BMI $\left(\mathrm{kg} / \mathrm{m}^{2}\right)$ & 1.044 (0.979 to 1.112$)$ & 0.19 & 1.005 (0.991 to 1.020$)$ & 0.49 & 0.999 (0.994 to 1.005$)$ & 0.83 \\
\hline Prewash TPMSC (M) & 0.998 (0.993 to 1.004$)$ & 0.55 & 1.002 (0.999 to 1.004$)$ & 0.16 & 0.995 (0.987 to 1.002$)$ & 0.15 \\
\hline Postwash TPMSC (M) & 1.005 (0.998 to 1.012$)$ & 0.14 & $1.000(0.998$ to 1.001$)$ & 0.7 & $1.010(1.002$ to 1.017$)$ & 0.01 \\
\hline
\end{tabular}

BMI, body mass index; FSH, follicle-stimulating hormone; TPMSC, total progressive motile sperm count. 
A

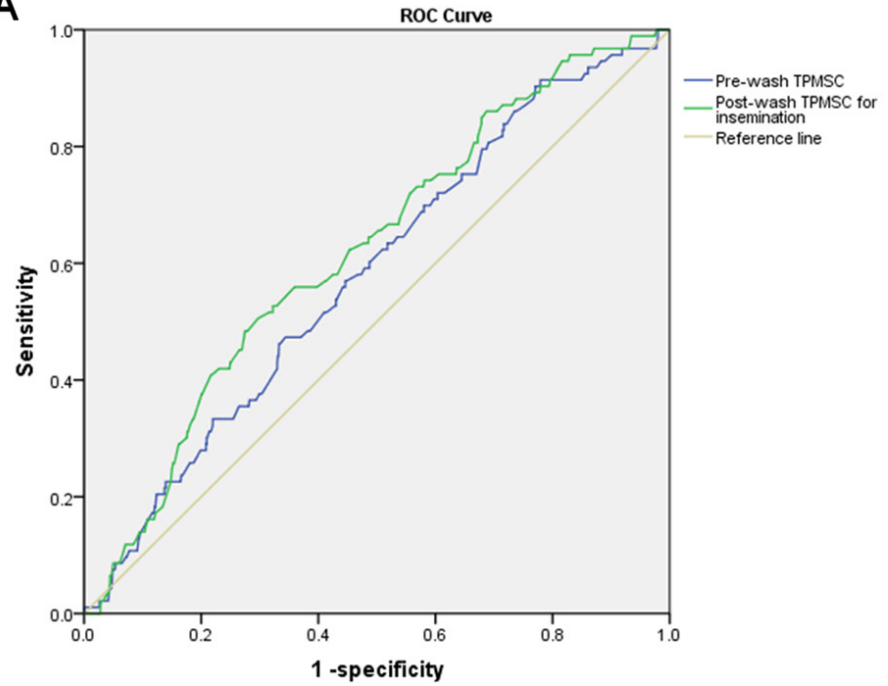

B

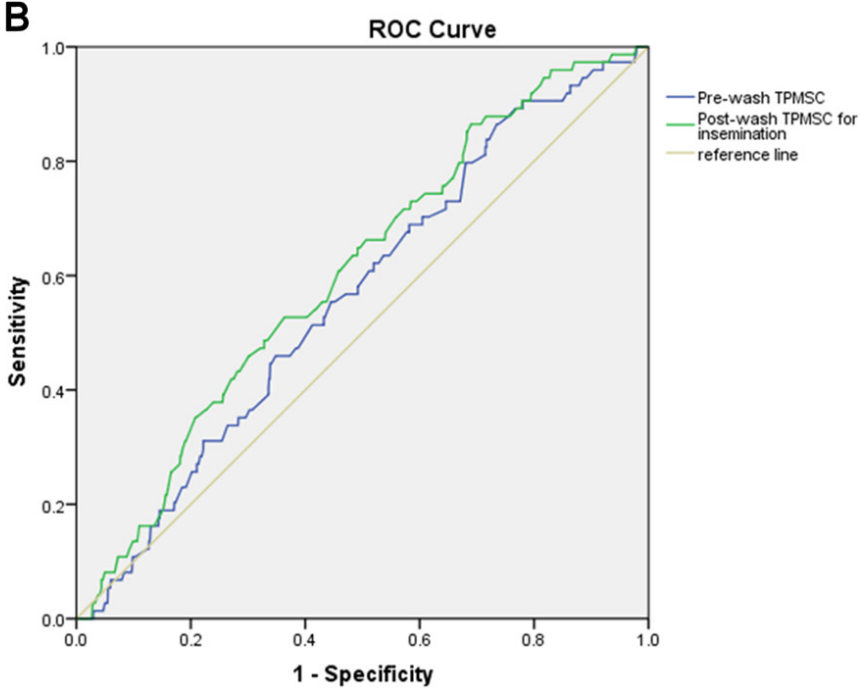

Figure 2 ROC curve of prewash and postwash TPMSCs with clinical pregnancy and live birth in mild male factor infertility. (A) Prewash TPMSC: AUC 0.578, 95\% Cl 0.520 to 0.635, $p=0.012$; postwash TPMSC AUC 0.621, 95\% Cl 0.564 to $0.677, p<0.001$. When postwash TPMSC was $55.40 \mathrm{M}$, it can predict clnical pregnancy with a sensitivity of $50 \%$ and a specificity of $71 \%$. (B) Prewash TPMSC: AUC 0.565, 95\% Cl 0.502 to 0.628, p=0.063; postwash TPMSC AUC 0.605, 95\% Cl 0.543 to $0.667, p=0.002$. When postwash TPMSC was $15.37 \mathrm{M}$, it can predict live birth with a sensitivity of $86.5 \%$ and a specificity of $31.0 \%$. AUC, area under the curve; ROC, receiver operating characteristic; TPMSC, total progressive motile sperm count.

Unexplained infertility, mild male factor and ovulation disorders are the main causes of infertility referred to IUI treatment. Transferring high-quality and quantity motile sperms into the uterine cavity is the main goal of an IUI procedure. Until now, only a few studies explored the relationship between TMSC and unexplained infertility and mild male factor infertility in IUI. ${ }^{21} 22$

IUI increasing the concentration of motile sperms in the uterus is a prevalent procedure as a low-cost and less invasive approach for infertile couples, and it has been reported that the CPR of IUI varies between $8 \%$ and $22 \%,{ }^{129}$ mainly due to different infertility factors. In a previous study on IUI in the Netherlands, patient characteristics 'diagnosis' and 'female age' determined the outcome variation. The authors proposed that the identification of a suitable patient population in IUI is important to tailor interventions which might improve the results of IUI. ${ }^{30}$ Our results corroborate this statement as patients with oligo-ovulation/anovulation benefitted most from IUI, and patients with unexplained infertility had the poorest results after IUI.

Treatment of unexplained infertility theoretically is difficult. Isa $e t$ al showed that the CPR in cases of unexplained infertility was $8.45 \% .{ }^{20}$ However, others concluded that IUI can be performed as first-line treatment in unexplained infertility, providing an acceptable pregnancy rate $(>10 \%) .{ }^{22}$ Moreover, Ashrafi et al, in primary infertile patients with less than 5 years of infertility and an inseminated motile sperm count of $>30$ million, reported ${ }^{23}$ a CPR per cycle of $19.9 \% .{ }^{2023}$ In contrast, from our analysis, it seems that the efficacy of IUI in unexplained infertility is quite limited, as the CPR and LBR were less than $10 \%$ per cycle.
Until now, no consensus exists regarding the semen parameters for IUI. Some authors define the concentration as sperm concentration per millilitre or as the prewash total number of motile sperm or as postwash total number of motile sperm. ${ }^{5611} 123132$ It has been reported that pregnancy rates are lower if the total motile sperm count in the ejaculate is less than 10 million. ${ }^{33}$ The lower limit ranges from 3 million motile sperms to 5 million-10 million for insemination.

The intention of the present analysis based on a large sample size was to explore the optimal range in different infertility diagnoses. TPMSC cannot be solely taken as a predictor in clinical pregnancy. Interestingly, it was reported that in mild male factor infertility, postwash TPMSC is closely associated with clinical pregnancy and LBR. We conclude that prewash TPMSC $>75 \mathrm{M}$ and postwash TPMSC $>65.10 \mathrm{M}$ result in an acceptable CPR and LBR in mild male factor infertility. From the reported semen analyses of consecutive semen samples, consecutive ejaculates can be used to improve the semen quality in IUI cycles. ${ }^{34}$

Female age is an independent factor for clinical pregnancy and live birth, although a study by Isa $e t$ al found no association between pregnancy rate and age. ${ }^{20}$ However, many studies have reported a significant decrease in CPR beyond the age of 40 years, with reported live births being as low as $1.4 \% .{ }^{35}$ It is reported that pregnancy rates decreased after women aged 30 years and significantly decreased after women aged 40 years. ${ }^{23}$ According to their analysis, no IUI should be suggested to women older than 40 years. Due to the relatively small sample size of women older than 40 years we cannot set firm conclusions from our data set.

In future, it would be helpful and practical for couples and clinicians if a prediction model for IUI could be made. 
Through the ROC curve and logistic regression analysis in our study, prewash and postwash TPMSCs independently were poor indicators for LBR. However, in separate patients with mild male factor infertility, the postwash TPMSC showed a moderate power to predict CPR.

The limitation of the present study is its retrospective nature, and other factors influencing the IUI success rate are not considered. Moreover, although semen analyses did not change during recent years, the data from a single centre may not be directly applicable to other settings and areas of the world.

In conclusion, based on couple characteristics like age, infertility factors, TPMSC and previous postwash TPMSC, the IUI treatment plan needs to be individualised. We suggest IUI should be provided mainly for patients with ovulation disorders. For patients with unexplained infertility, TPMSC has little impact, and the benefit from IUI treatment may be very limited. For patients with mild male factor infertility, the TPMSC should be taken into account for the outcome. Overall, in couples with female age more than 40 years, IUI should not be performed.

Contributors HL and YL planned, designed and drafted the study; SO was responsible for the data collection; $\mathrm{XJ}$ conducted the main part of the analysis: WW and QZ contributed to the interpretation of the analysis. PH and QZ revised the manuscript. All authors approved the final version.

Funding This study was funded by National Natural Science Funding (number 81701519).

\section{Competing interests None declared.}

Patient and public involvement Patients and/or the public were not involved in the design, conduct, reporting or dissemination plans of this research.

\section{Patient consent for publication Not required.}

Ethics approval The study was approved by the ethics committee of Sun Yat-sen Memorial Hospital (number SYSEC-KY-KS-2019-162).

Data availability statement The data that support the findings of this study are available on request from the corresponding author.

Open access This is an open access article distributed in accordance with the Creative Commons Attribution Non Commercial (CC BY-NC 4.0) license, which permits others to distribute, remix, adapt, build upon this work non-commercially, and license their derivative works on different terms, provided the original work is properly cited, appropriate credit is given, any changes made indicated, and the use is non-commercial. See: http://creativecommons.org/licenses/by-nc/4.0/.

ORCID iD

Haiyan Lin http://orcid.org/0000-0002-5771-3947

\section{REFERENCES}

1 Ferraretti AP, Goossens V, Kupka M, et al. Assisted reproductive technology in Europe, 2009: results generated from European registers by ESHRE. Hum Reprod 2013;28:2318-31.

2 Bai F, Wang DY, Fan YJ, et al. Assisted reproductive technology service availability, efficacy and safety in mainland China: 2016. Hum Reprod 2020;35:446-52.

3 Bahadur G, Homburg R, Bosmans JE, et al. Observational retrospective study of UK national success, risks and costs for $319,105 \mathrm{IVF} / \mathrm{ICSI}$ and 30,669 IUI treatment cycles. BMJ Open 2020;10:e034566.

4 Duca Y, Calogero AE, Cannarella R, et al. Current and emerging medical therapeutic agents for idiopathic male infertility. Expert Opin Pharmacother 2019;20:55-67.

5 Hamilton JAM, Cissen M, Brandes M, et al. Total motile sperm count: a better indicator for the severity of male factor infertility than the WHO sperm classification system. Hum Reprod 2015;30:1110-21.
6 Hajder M, Hajder E, Husic A. The effects of total motile sperm count on spontaneous pregnancy rate and pregnancy after iui treatment in couples with male factor and unexplained infertility. Med Arch 2016;70:39-43.

7 Ombelet W, Dhont N, Thijssen A, et al. Semen quality and prediction of IUI success in male subfertility: a systematic review. Reprod Biomed Online 2014;28:300-9.

8 Deveneau NE, Sinno O, Krause M, et al. Impact of sperm morphology on the likelihood of pregnancy after intrauterine insemination. Fertil Steril 2014;102:1584-90.

9 Merviel P, Heraud MH, Grenier N, et al. Predictive factors for pregnancy after intrauterine insemination (IUI): an analysis of 1038 cycles and a review of the literature. Fertil Steril 2010;93:79-88.

10 Pereira N. Total motile count as predictor of live birth in intrauterine insemination cycles. Fertil Steril 2019;111:674.

11 Mankus EB, Holden AE, Seeker PM, et al. Prewash total motile count is a poor predictor of live birth in intrauterine insemination cycles. Fertil Steril 2019;111:708-13.

12 Patounakis G, Richter KS, DeCherney A, et al. Pre-wash sperm morphology predicts IUI outcomes by predicting post-wash total motile sperm count (TMSC): an analysis of 26,373 IUI cycles. Fertil Steril 2015;104:e239-40.

13 Bensdorp A CBJ, Heineman MJ, et al. Intra-uterine insemination for male subfertility. Cochrane Database System Rev 2007;4.

14 Bhandari S, Ganguly I, Agarwal P, et al. The dilemma of unexplained infertility: laparoscopy versus intrauterine insemination. IVF Lite 2015;2:88-93.

15 Tjon-Kon-Fat RI, Tajik P, Zafarmand $\mathrm{MH}$, et al. IVF or IUI as first-line treatment in unexplained subfertility: the conundrum of treatment selection markers. Hum Reprod 2017;32:1028-32.

16 Practice Committee of the American Society for Reproductive Medicine. Effectiveness and treatment for unexplained infertility. Fertil Steril 2006;86:S111-4.

17 O'Flynn N, O'Flynn N. Assessment and treatment for people with fertility problems: NICE guideline. Br J Gen Pract 2014;64:50-1.

18 Haagen EC, Nelen WLDM, Adang EM, et al. Guideline adherence is worth the effort: a cost-effectiveness analysis in intrauterine insemination care. Hum Reprod 2013;28:357-66.

19 Kim D, Child T, Farquhar C. Intrauterine insemination: a UK survey on the adherence to NICE clinical guidelines by fertility clinics. BMJ Open 2015;5:e007588.

20 Isa AM, Abu-Rafea B, Alasiri SA, et al. Accurate diagnosis as a prognostic factor in intrauterine insemination treatment of infertile Saudi patients. J Reprod Infertil 2014;15:184-9.

21 Atasever M, Kalem MN, Hatırnaz Şafak, et al. Factors affecting clinical pregnancy rates after IUI for the treatment of unexplained infertility and mild male subfertility. J Turk Ger Gynecol Assoc 2016;17:134-8.

22 Ganguly I, Singh A, Bhandari S, et al. Pregnancy predictors after intrauterine insemination in cases of unexplained infertility: a prospective study. Int J Reprod Med 2016;2016:5817823:1-5.

23 Ashrafi M, Rashidi M, Ghasemi A, et al. The role of infertility etiology in success rate of intrauterine insemination cycles: an evaluation of predictive factors for pregnancy rate. Int J Fertil Steril 2013;7:100-7.

24 Sinha P, Pandey K, Srivastava A. Factors determining successful intrauterine insemination. Int J Reprod Contracept Obstet Gynecol 2017;6:3887.

25 Tatsumi T, Ishida E, Tatsumi K, et al. Advanced paternal age alone does not adversely affect pregnancy or live-birth rates or sperm parameters following intrauterine insemination. Reprod Med Biol 2018;17:459-65.

26 Thijssen A, Creemers A, Van der Elst W, et al. Predictive factors influencing pregnancy rates after intrauterine insemination with frozen donor semen: a prospective cohort study. Reprod Biomed Online 2017;34:590-7.

27 Bahadur G, Woodward B, Homburg R, et al. Pitfalls of NICE recommendations on fertility treatment. BMJ 2017;356:j751.

28 WHO. WHO laboratory manual for the examination and processing of human semen, 2010.

29 Bagis T, Haydardedeoglu B, Kilicdag EB, et al. Single versus double intrauterine insemination in multi-follicular ovarian hyperstimulation cycles: a randomized trial. Hum Reprod 2010;25:1684-90.

30 Hermens RPMG, Haagen EC, Nelen WLDM, et al. Patient and hospital characteristics associated with variation in guideline adherence in intrauterine insemination care. Int J Qual Health Care 2011;23:574-82.

31 Lemmens L, Kos S, Beijer C, et al. Predictive value of sperm morphology and progressively motile sperm count for pregnancy outcomes in intrauterine insemination. Fertil Steril 2016;105:1462-8. 
32 Rubin RS, Richter KS, Naeemi F, et al. Redefining and clarifying the relationship between total motile sperm counts (TMSC) and intrauterine insemination (IUI) pregnancy rates. Fertil Steril 2015;104:e9.

33 Van Voorhis BJ, Barnett M, Sparks AE, et al. Effect of the total motile sperm count on the efficacy and cost-effectiveness of intrauterine insemination and in vitro fertilization. Fertil Steril 2001;75:661-8.
34 Bahadur G, Almossawi O, Zeirideen Zaid R, et al. Semen characteristics in consecutive ejaculates with short abstinence in subfertile males. Reprod Biomed Online 2016;32:323-8.

35 Tay PYS, Raj VRM, Kulenthran A, et al. Prognostic factors influencing pregnancy rate after stimulated intrauterine insemination. Med $\mathrm{J}$ Malaysia 2007;62:286-9.

36 Ibérico G, Vioque J, Ariza N, et al. Analysis of factors influencing pregnancy rates in homologous intrauterine insemination. Fertil Steril 2004;81:1308-13. 